\title{
Diversidade de Cupins em Áreas de Savana, Submetidas a Diferentes Regimes de Fogo
}

\author{
Otávio Peres Filho ${ }^{1}$, Keila Sandra de Oliveira ${ }^{1}$, \\ Marcelo Dias de Souza ${ }^{2}$, Alberto Dorval ${ }^{1}$ \\ ${ }^{1}$ Departamento de Engenharia Florestal, Universidade Federal de Mato Grosso - UFMT \\ ${ }^{2}$ Programa de Pós-Graduação em Ciências Florestais e Ambientais, Universidade Federal de Mato Grosso - UFMT
}

\begin{abstract}
RESUMO
O trabalho teve como objetivos coletar, identificar e quantificar as espécies de cupins que ocorrem em três áreas de savana submetidas à ação do fogo em diferentes épocas. O estudo foi realizado no município de Cuiabá, estado de Mato Grosso, no período de maio de 2004 a março de 2005. Foram amostradas três áreas distintas quanto à ação do fogo: área 1 - um ano sem ocorrência de fogo; área 2-10 anos sem ocorrência de fogo; área 3-17 anos sem a ocorrência de fogo. Foram utilizadas 20 armadilhas por área, modelo Termitrap. As armadilhas foram dispostas em forma de cruz, permanecendo enterradas no solo por 15 dias a cada período de coleta. As amostras foram levadas ao laboratório, onde foram triadas, quantificadas e acondicionadas em álcool 70\%, para identificação. Foram coletados 167.316 indivíduos, distribuídos em 13 espécies, sendo a espécie Heterotermes tenuis a mais representativa quantitativamente nas áreas estudadas. Os cupins apresentaram preferência significativa pela área com 10 anos sem a ausência de fogo e o período de coleta no início das chuvas foi significativamente maior em quantidade de indivíduos, porém com menor quantidade de espécies.
\end{abstract}

Palavras-chave: isoptera, cerrado, ecologia do fogo, Termitrap.

\section{Diversity of Termites in Savanna Areas Submitted to Different Fire Activities}

\begin{abstract}
The purpose of this research was to collect, identify and quantify species of termite in three savanna areas submitted to different fire activities in different seasons. The study was carried out in Cuiaba, State of Mato Grosso, between May 2004 and March 2005. Samples were collected from three different areas submitted to fire: area $1-1$ year without fire occurrence; area 2-10 years without fire occurrence; area 3-17 years without occurrence fire. Twenty Termitrap model traps were used per area. The traps were installed in cross form, staying buried in the soil for 15 days at each collection period. The samples were transported to the laboratory where they were separated, quantified and conditioned in alcohol $70 \%$ for identification. The collection resulted in 167.316 individuals of 13 species. Heterotermes tenuis was the most quantitatively representative species in the studied areas. The termites showed significant preference for area 2-10 years without fire occurrence. The collection period in the beginning of the rain season yielded a significantly greater amount of individuals, but fewer species.
\end{abstract}

Keywords: isoptera, cerrado, ecology of fire, Termitrap. 


\section{INTRODUÇÃO}

O cerrado é susceptível à ocorrência de queimadas, especialmente durante o período dos meses de baixa precipitação pluviométrica, quando a cobertura do solo, geralmente composta por gramíneas, propicia condições favoráveis para a ocorrência de grandes focos de incêndios; estes podem causar modificações diretas e indiretas no ambiente, afetando a abundância e a diversidade de várias espécies de insetos.

O fogo é uma das mais frequentes e importantes causas de distúrbio no cerrado, com efeitos de curto e longo prazo. Os efeitos de longo prazo sobre as comunidades animais são muito pouco conhecidos (Vianna et al., 2004). Estudos da entomofauna desse bioma têm fornecido informações importantes sobre as alterações ambientais de forma rápida e confiável. Os cupins, dentre outros grupos de insetos, têm sido utilizados como bioindicadores dessas alterações ocasionadas por fatores naturais ou antrópicos (Espírito-Santo Filho, 2005).

Os cupins são organismos particularmente evidentes e abundantes no cerrado, onde alguns tipos de cupinzeiros podem alcançar elevados níveis de densidade. $\mathrm{Na}$ Amazônia, embora também apresentem grande diversidade e abundância, não são tão evidentes como no cerrado (Constantino, 1999). Vários estudos relatam que os cupins são diretamente afetados pelas alterações antrópicas e pelas devastações do cerrado por práticas agrícolas, tornando-se pragas em razão dos desequilíbrios ecológicos. Porém, desempenham papel ecológico importante em um ecossistema natural, pois são decompositores e atuam na ciclagem e no transporte de nutrientes do solo (Lobry \& Conacher, 1990).

Apesar dos cupins serem muito estudados, há falta de informações sobre a abundância, a distribuição e a diversidade das espécies existentes no cerrado (Constantino, 2005). Entre as perturbações naturais que a vegetação do cerrado sofre periodicamente, o fogo é uma das mais importantes (Coutinho, 1980). O cerrado é susceptível às queimadas, especialmente durante a estação seca, quando a camada rasteira, dominada por gramíneas, seca.

Almeida \& Alves (1995) desenvolveram um tipo de isca artificial registrada como Termitrap ${ }^{\circledast}$ (rolo de papelão ondulado). Essa isca vem sendo utilizada em monitoramentos, substituindo os levantamentos convencionais de arranquio de soqueiras ou plantio de mudas de cana-de-açúcar como iscas (Campos et al., 1998). A isca artificial registrada como Termitrap (rolo de papelão corrugado) tem sido utilizada para o monitoramento e o controle de alguns cupins de solo, onde tem proporcionado ótimos resultados no controle de áreas infestadas, pois permite a localização dos focos, tornando o controle mais econômico e ecológico, além de demonstrarem a sua eficiência na atração de cupins dos gêneros Cornitermes, Syntermes, Procornitermes, Coptotermes e Nasutitermes. (Almeida \& Alves, 1995).

Outra importante característica dessa armadilha é o seu baixo custo, o alto teor de celulose e a facilidade na aquisição (French et al., 1981; Sueta, 1984; Jones et al., 1987). Portanto, o presente estudo teve como objetivo analisar qualitativa e quantitavamente as espécies de cupins que ocorrem em três áreas de savana, submetidas à ação do fogo em diferentes épocas, utilizando-se a isca Termitrap.

\section{MATERIAL E MÉTODOS}

A pesquisa foi realizada em uma área de Savana Arbórea Aberta com 48 ha do Instituto de Linguística de Mato Grosso, localizada entre as coordenadas geográficas $15^{\circ} 39^{\prime} 723^{\prime \prime} \mathrm{S}$ e $56^{\circ} 00.638^{\prime} \mathrm{W}$, no município de Cuiabá, estado de Mato Grosso. Os tipos de solos predominantes do local são o concrecionário distrófico e o podzólico vermelho-amarelo distrófico (Brasil, 1982). O clima é tropical continental (Aw), caracterizando-se por um inverno seco e um verão chuvoso. A temperatura média anual é de $25,6^{\circ} \mathrm{C}$ e a precipitação anual é de $1421 \mathrm{~mm}$, com 95\% ocorrendo entre os meses de outubro e março.

Para as amostragens da termitofauna, foram utilizadas armadilhas Termitrap, com as dimensões de $72 \mathrm{~cm} \times 18 \mathrm{~cm}$, enroladas e presas por barbante na forma cilíndrica como um "rocambole", com $6 \mathrm{~cm}$ de diâmetro. As armadilhas foram instaladas em três áreas, sendo um hectare para cada uma e de acordo com a seguinte distribuição: $\mathrm{A} 1$ = área com um ano sem fogo; A2 = área com dez anos sem fogo, 
e A3 = área com mais de 17 anos sem fogo. Em cada área, foram implantadas 20 armadilhas, totalizando 60 armadilhas em cada período de coleta, tendo sido analisados três períodos:

- Primeiro período: Maio de 2004 (período seco);

- Segundo período: Outubro de 2004 (início do período de chuva);

- Terceiro período: Março 2005 (final do período chuvoso).

As armadilhas foram dispostas em forma de cruz, distribuídas com dez metros de distância entre si (Figura 1). As armadilhas foram umedecidas com água e enterradas verticalmente com a extremidade superior tangenciando a superfície do solo, permanecendo por um período de 15 dias no campo. Após esse período, foram retiradas e devidamente embaladas, identificadas com o número da armadilha e a data da coleta, e foram então transportadas para o Laboratório de Proteção Florestal, da Faculdade de Engenharia Florestal, da Universidade Federal de Mato Grosso.

Os exemplares coletados foram separados das impurezas pelo método de flotação, os espécimes coletados foram quantificados e, posteriormente, acondicionados em frascos de $15 \mathrm{ml}$ contendo álcool $70 \%$, sendo enviados ao departamento de Zoologia da Universidade de Brasília-UnB, para identificação. Os dados obtidos de cada área foram submetidos

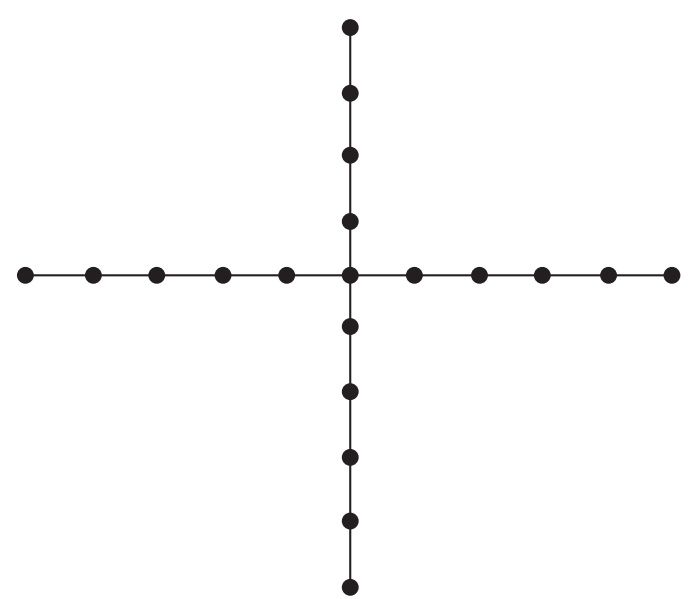

Figura 1. Demonstração esquemática das disposições das armadilhas em forma de cruz, distribuídas a $10 \mathrm{~m}$ de distância entre si. Cuiabá-MT, 2004-2005.

Figure 1. Schematic demonstration of the traps spreads in form of cross, distributed in $10 \mathrm{~m}$ of distance among themselves. Cuiabá, State of Mato Grosso, 2004-2005. às análises faunísticas de dominância de Sakagami \& Laroca (1967), de abundância, frequência e constância, utilizando-se o software ANAFAU (Moraes et al., 2003).

Nas análises estatísticas, foram realizados dois delineamentos inteiramente casualizados em esquema fatorial (Espécies $\times$ Área; Espécie $\times$ Período de coleta), com cinco repetições (considerando-se quatro armadilhas como uma repetição), cujos dados foram submetidos à análise de variância (ANOVA) e as médias foram comparadas pelo teste de ScottKnott no nível de 5\% de significância. Levando-se em conta a distribuição dos valores da quantidade de cupins nas três áreas de savana, utilizou-se a transformação dos dados com a fórmula: $\sqrt{X+0,5}$ , para a uniformização e normalidade (Phillips, 1990). As análises foram executadas utilizando-se o software SISVAR (Ferreira, 2008).

\section{RESULTADOS E DISCUSSÃO}

Foram coletados 167.316 indivíduos, distribuídos em 13 espécies. A família Termitidae com 11 espécies foi quantitativamente a mais representativa, enquanto Rhinotermitidae foi a mais importante em número de indivíduos coletados; a espécie Heterotermes tenuis foi a mais quantitativamente representativa, com 89.202 indivíduos coletados, correspondendo a $53,31 \%$ do total de espécimes coletados (Tabela 1). Resultado semelhante foi obtido por Santos (2004), em plantios de Eucalyptus camaldulensis Dehnh no município de Várzea Grande/MT, onde as espécies H. tenuis e Nasutitermes kemneri (Snyder \& Emerson, 1949) foram quantitativamente as mais representativas.

Os resultados obtidos por Vianna et al. (2004) sobre o efeito do fogo em cupins subterrâneos no cerrado corroboram os resultados obtidos. Foram estudadas duas áreas, uma com cerca de 30 anos protegida do fogo e outra que tinha sofrido a ação do fogo recentemente, constatando-se a ocorrência dos gêneros Heterotermes, Nasutitermes, Velocitermes e Diversitermes. Ruptitermes sp. foi quantitativamente a menos expressiva, com 75 indivíduos, correspondendo a $0,04 \%$ do total de espécimes coletados. 
Tabela 1. Família, espécies, quantidade e percentual de cupins coletados com armadilhas “Termitrap” em áreas de savana. Cuiabá-MT, 2004-2005.

Table 1. Families, species, quantity and percentage of termites collected with Termitrap traps in savanna areas. Brazil, Cuiabá, State of Mato Grosso, 2004-2005.

\begin{tabular}{|lrc|}
\hline \multicolumn{1}{|c}{ FAMÍLIA/espécie } & Quantidade & (\%) \\
\hline TERMITIDAE & & 0,23 \\
\hline Anoplotermes sp. & 385 & 0,49 \\
\hline Cornitermes silvestrii Emerson, 1952 & 822 & 1,42 \\
\hline Cornitermes villosus Emerson, 1952 & 2.368 & 0,10 \\
\hline Cylindrotermes parvignathus Snyder, 1949 & 164 & 8,90 \\
\hline Diversitermes diversimiles (Silvestri, 1901) & 14.890 & 29,53 \\
\hline Nasutitermes kemneri Snyder, 1949 & 49.395 & 0,56 \\
\hline Parvitermes bacchanalis Mathews, 1977 & 939 & 0,04 \\
\hline Ruptitermes sp. & 75 & 0,38 \\
\hline Syntermes nanus Constantino, 1995 & 638 & 3,90 \\
\hline Velocitermes heteropterus (Silvestri, 1901) & 6.531 & 0,54 \\
\hline Não identificada) & 902 & 0,60 \\
\hline RHINOTERMITIDAE & & 53,31 \\
\hline Heterotermes longiceps Snyder, 1924 & 1.005 & 100,00 \\
\hline Heterotermes tenuis (Hagen, 1858) & 89.202 & 167.316 \\
\hline Total
\end{tabular}

Na área 1, ocorreram sete espécies, com 38.912 $(23,26 \%)$ dos indivíduos. Quantitativamente, quando comparada com as demais áreas amostradas, a área 1 foi menos representativa e tal fato, talvez, deva-se à presença constante do fogo, que pode ter afetado diretamente a população desses insetos ou indiretamente, na disponibilidade de alimento (Tabela 2). Na área 2, foram coletadas dez espécies, com 86.068 indivíduos (51,44\%); já na área 3, embora esta representasse o local com o maior período sem ocorrência do fogo, foi registrada uma menor quantidade de espécies (9) e de indivíduos $42.336(25,30 \%)$.

Esse fato, talvez, possa estar correlacionado com uma maior estabilidade das condições ambientais nessa área, propiciando às espécies condições favoráveis para o estabelecimento e o desenvolvimento de suas colônias. As espécies Diversitermes diversimiles, $H$. tenuis, N. kemneri, Parvitermes bacchanalis e Velocitermes heteropterus ocorreram indistintamente nas três áreas. Cylindrotermes parvignathus e Ruptitermes sp. ocorreram somente na área 2 e Heterotermes longiceps ocorreu somente na área 3; todas estas, no período seco, o que pode estar correlacionado com a maior estabilidade ambiental em razão da ausência de fogo, podendo-se admitir a hipótese de que essas espécies sejam bioindicadoras de ambientes mais equilibrados.

$\mathrm{Na}$ primeira coleta realizada no período seco, foram observados 63.108 indivíduos, correspondendo a $37,72 \%$ do total de espécimes. Na segunda, realizada no início do período chuvoso, foram coletados 72.488 indivíduos (43,32\%). A terceira coleta, realizada no final do período chuvoso, foi quantitativamente a menos expressiva, com 31.720 ou $18,96 \%$ do total coletado nos três períodos analisados (Tabela 3 ).

Provavelmente, a menor quantidade de indivíduos coletados no final do período chuvoso esteja relacionada à saturação do solo pela umidade, o que pode ter provocado uma maior mortalidade, considerando-se que o solo da área apresenta pouca profundidade. Esses dados diferem dos obtidos por Santos (2004), que coletou uma maior quantidade de indivíduos no final do período chuvoso.

Nos estudos faunísticos de frequência, na análise geral em ambas as áreas, observou-se que duas espécies ocorreram como pouco frequentes, cinco frequentes, uma muito frequente e quatro super 
Tabela 2. Quantidade de indivíduos por espécie, coletados nas três áreas de savana estudadas. Cuiabá-MT, 20042005.

Table 2. Amount of individuals by specie, collected in the three areas of savanna studied. Brazil, Cuiabá, State of Mato Grosso, 2004-2005.

\begin{tabular}{lccc} 
& \multicolumn{1}{c}{ Espécie } & Área & \\
\cline { 2 - 4 } & $\mathbf{1}$ & $\mathbf{2}$ & $\mathbf{3}$ \\
Anoplotermes sp. & 15 & - & 370 \\
Cornitermes silvestrii & 21 & 801 & - \\
\hline Cornitermes villosus & - & 164 & 4 \\
Cylindrotermes parvignathus & - & 3.777 & 5.996 \\
Diversitermes diversimiles & 5.117 & - & 1.005 \\
Heterotermes longiceps & - & 45.086 & 23.057 \\
Heterotermes tenuis & 21.059 & 28.381 & 11.150 \\
Nasutitermes kemneri & 9.864 & 191 & 71 \\
\hline Parvitermes bacchanalis & 677 & 75 & - \\
Ruptitermes sp. & - & 557 & 81 \\
Syntermes nanus & - & 3.777 & 595 \\
Velocitermes heteropterus & 2.159 & 895 & 7 \\
Nasutitermitinae (Não identif.) & - & 86.068 & 42.336 \\
Total & 38.912 & 51,44 & 25,30 \\
\hline (\%) & 23,26 & & - \\
\hline
\end{tabular}

Tabela 3. Quantidade de indivíduos por espécie, coletados em três períodos de coletas nas três áreas de savana. Cuiabá-MT, 2004-2005.

Table 3. Amount of individuals by specie, collected in the three periods in the areas of savanna studied. Brazil, Cuiabá, State of Mato Grosso, 2004-2005.

\begin{tabular}{lccc} 
& \multicolumn{1}{c}{ Espécie } & Coletas & Chuvoso \\
\cline { 2 - 4 } & Seco & Início das chuvas & 50 \\
Anoplotermes sp. & 335 & - & 21 \\
Cornitermes silvestrii & 11 & 790 & 146 \\
Cornitermes villosus & 624 & 1598 & - \\
Cylindrotermes parvignathus & 164 & - & 8204 \\
\hline Diversitermes diversimiles & 4680 & 2006 & - \\
\hline Heterotermes longiceps & 1005 & - & 19667 \\
Heterotermes tenuis & 29387 & 40148 & 2261 \\
Nasutitermes kemneri & 22353 & 24781 & 125 \\
Parvitermes bacchanalis & 623 & 191 & - \\
Ruptitermes sp. & 75 & - & 238 \\
Syntermes nanus & 16 & 384 & 1008 \\
Velocitermes heteropterus & 3828 & 1695 & - \\
\hline Nasutitermitinae (Não identif.) & 7 & 895 & 31.720 \\
\hline Total & 63.108 & 72.488 & 18,96 \\
\hline (\%) & 37,72 & 43,32 & \\
\hline
\end{tabular}

frequentes; quanto à constância, observou-se que nove espécies ocorreram como constantes e três como acessórias, enquanto cinco espécies foram dominantes e quatro não dominantes (Tabela 4).

Quanto à abundância, duas espécies ocorrem como raras, cinco comuns, uma muito abundante e quatro superabundantes. Oliveira et al. (2003) obtiveram resultados semelhantes estudando a variação na composição de espécies e no padrão de riqueza e abundância de cupins ao longo de uma transição cerrado-campo rupestre no município de Hidrolândia, estado de Goiás, onde observaram que 
Tabela 4. Índices faunísticos das espécies de cupins coletadas com armadilhas Termitrap em áreas de savana em todo o período avaliado. Cuiabá, MT, 2004-2005.

Table 4. Faunistics index of the species of termites collected with Termitrap traps in savanna areas in all period available. Brazil, Cuiabá, State of Mato Grosso, 2004-2005.

\begin{tabular}{|c|c|c|c|c|}
\hline \multirow{2}{*}{ Espécies } & \multicolumn{4}{|c|}{ Índices faunísticos } \\
\hline & $\mathbf{F}$ & A & $\mathrm{C}$ & D \\
\hline Anoplotermes sp. & $\mathrm{f}$ & c & $\mathrm{w}$ & nd \\
\hline Cornitermes silvestrii & $\mathrm{f}$ & c & $\mathrm{w}$ & $\mathrm{d}$ \\
\hline Cornitermes villosus & $\mathrm{mf}$ & $\mathrm{ma}$ & $\mathrm{w}$ & d \\
\hline Cylindrotermes parvignathus & $\mathrm{pf}$ & $r$ & $\mathrm{y}$ & nd \\
\hline Diversitermes diversimiles & sf & sa & $\mathrm{w}$ & sd \\
\hline Heterotermes longiceps & $\mathrm{f}$ & c & $\mathrm{y}$ & $\mathrm{d}$ \\
\hline Heterotermes tenuis & sf & sa & $\mathrm{w}$ & sd \\
\hline Nasutitermes kemneri & sf & sa & $\mathrm{w}$ & sd \\
\hline Parvitermes bacchanalis & $\mathrm{f}$ & c & $\mathrm{w}$ & $\mathrm{d}$ \\
\hline Ruptitermes sp. & $\mathrm{pf}$ & $\mathrm{r}$ & $\mathrm{y}$ & nd \\
\hline Syntermes nanus & $\mathrm{f}$ & c & $\mathrm{w}$ & nd \\
\hline Velocitermes heteropterus & sf & sa & $\mathrm{w}$ & sd \\
\hline Nasutitermitinae (Não identif.) & $\mathrm{f}$ & $c$ & $\mathrm{w}$ & $\mathrm{d}$ \\
\hline
\end{tabular}

F: frequência - $(\mathrm{pf})$ pouco frequente, $(\mathrm{f})$ frequente, $(\mathrm{mf})$ muito frequente, $(\mathrm{sf})$ super frequente; $\mathrm{A}$ : abundância - $(\mathrm{r})$ rara, $(\mathrm{c})$ comum, (ma) muito abundante, (sa) super abundante; C: constância - (w) constante, (y) acessória; D: dominância - (d) dominante, (nd) não dominante, (sd) super dominante.

as espécies Anoplotermes sp. (61 colônias), N. kemneri (51 colônias) e $V$. heteropterus (31 colônias) foram as mais abundantes.

$\mathrm{Na}$ área de savana com um ano sem a presença de fogo, ocorreram cinco espécies frequentes, uma muito frequente e uma super frequente. Em relação à dominância, duas espécies apresentaram-se como dominantes, uma superdominante e quatro não dominantes. Já na área com dez anos sem presença de fogo, ocorreram três espécies pouco frequentes, três frequentes, duas muito frequentes e duas super frequentes; quanto à dominância, constataram-se três dominantes, duas superdominantes e cinco não dominantes (Tabela 5).

$\mathrm{Na}$ área de savana com mais de 17 anos sem a presença de fogo, constataram-se uma espécie pouco frequente, três frequentes e duas muito frequentes. $\mathrm{Na}$ análise da abundância, uma espécie foi abundante, três comuns, uma muita abundante e três superabundantes. Em relação à constância, cinco espécies foram constantes e quatro acessórias. Quanto à dominância, três espécies foram dominantes, três superdominantes e três não dominantes. As espécies $D$. diversimiles, $H$. tenuis e $N$. kemneri ocorreram simultaneamente nas três áreas, sendo dominantes e superdominantes; já $P$. bacchanalis e $V$. heteropterus, apesar de serem encontradas em ambos os ambientes, não foram dominantes em todas as áreas estudadas.

A análise de variância apontou diferenças estatísticas entre as espécies coletadas nas três áreas estudadas, sendo que a espécie $H$. tenuis foi significativamente superior aos demais insetos, independentemente da área de ocorrência, apresentando preferência significativa pela área com dez anos sem ocorrência de fogo (Tabela 6). Em ambientes naturais, geralmente, $H$. tenuis é encontrado em madeira com certo grau de decomposição (Moino Junior et al., 2002) e, de acordo com Santos et al. (2010), é uma das principais pragas da cana-de-açúcar e do eucalipto no Brasil, ocorrendo principalmente nos estados do Sudeste e do Centro-Oeste do País.

Pode-se perceber que os térmitas encontrados nas três áreas apresentam preferência significativa pela área com dez anos sem a ausência de fogo, sendo a área com um ano sem a ausência de fogo a que revelou a menor preferência significativa, além de menor quantidade de espécies coletadas. Dias (1994), estudando o efeito do fogo sobre a diversidade de cupins, mostrou que algumas espécies 
Tabela 5. Índices faunísticos das espécies de cupins coletadas com armadilhas Termitrap nas três áreas de savana avaliada. Cuiabá-MT, 2004-2005.

Table 5. Faunistics index of the species of termites collected with Termitrap traps in the three savanna areas available. Brazil, Cuiabá, State of Mato Grosso, 2004-2005.

\begin{tabular}{|c|c|c|c|c|c|c|c|c|c|c|c|c|}
\hline \multirow{3}{*}{ Espécies } & \multicolumn{12}{|c|}{ Îndices faunísticos } \\
\hline & \multicolumn{4}{|c|}{ Área 1} & \multicolumn{4}{|c|}{ Área 2} & \multicolumn{4}{|c|}{ Área 3} \\
\hline & $\mathbf{F}$ & $\mathbf{A}$ & C & D & $\mathbf{F}$ & $\mathbf{A}$ & C & D & $\mathbf{F}$ & A & C & D \\
\hline Anoplotermes sp. & $\mathrm{f}$ & $\mathrm{ma}$ & $\mathrm{y}$ & nd & - & - & - & - & $\mathrm{f}$ & c & $\mathrm{w}$ & $\mathrm{d}$ \\
\hline Cornitermes silvestrii & $\mathrm{f}$ & $\mathrm{ma}$ & $\mathrm{y}$ & nd & $\mathrm{f}$ & c & $\mathrm{w}$ & nd & - & - & - & - \\
\hline Cornitermes villosus & - & - & - & - & $\mathrm{f}$ & c & $\mathrm{w}$ & $\mathrm{d}$ & $\mathrm{pf}$ & $\mathrm{d}$ & $\mathrm{y}$ & nd \\
\hline Cylindrotermes parvignathus & - & - & - & - & $\mathrm{pf}$ & d & $\mathrm{y}$ & nd & - & - & - & - \\
\hline Diversitermes diversimiles & $\mathrm{f}$ & $\mathrm{ma}$ & $\mathrm{w}$ & $\mathrm{d}$ & $\mathrm{mf}$ & $\mathrm{ma}$ & $\mathrm{w}$ & $\mathrm{d}$ & sf & sa & $\mathrm{w}$ & sd \\
\hline Heterotermes longiceps & - & - & - & - & - & - & - & - & $\mathrm{mf}$ & $\mathrm{ma}$ & $\mathrm{y}$ & $\mathrm{d}$ \\
\hline Heterotermes tenuis & sf & sa & $\mathrm{w}$ & sd & sf & sa & $\mathrm{w}$ & sd & sf & sa & $\mathrm{w}$ & sd \\
\hline Nasutitermes kemneri & $\mathrm{mf}$ & $\mathrm{ma}$ & $\mathrm{w}$ & $\mathrm{d}$ & sf & sa & $\mathrm{w}$ & sd & sf & sa & $\mathrm{w}$ & sd \\
\hline Parvitermes bacchanalis & $\mathrm{f}$ & $\mathrm{ma}$ & $\mathrm{w}$ & nd & $\mathrm{pf}$ & $\mathrm{d}$ & $\mathrm{y}$ & nd & $\mathrm{f}$ & c & $\mathrm{y}$ & nd \\
\hline Ruptitermes sp. & - & - & - & - & $\mathrm{pf}$ & $\mathrm{r}$ & $\mathrm{y}$ & nd & - & - & - & - \\
\hline Syntermes nanus & - & - & - & - & $\mathrm{f}$ & c & $\mathrm{w}$ & nd & $\mathrm{f}$ & c & $\mathrm{y}$ & nd \\
\hline Velocitermes heteropterus & $\mathrm{f}$ & $\mathrm{ma}$ & $\mathrm{w}$ & nd & $\mathrm{mf}$ & ma & $\mathrm{w}$ & $\mathrm{d}$ & $\mathrm{mf}$ & $\mathrm{a}$ & $\mathrm{w}$ & $\mathrm{d}$ \\
\hline
\end{tabular}

F: frequência - (pf) pouco frequente, (f) frequente, (mf) muito frequente, (sf) super frequente; A: abundância - (r) rara, (c) comum, (ma) muito abundante, (sa) super abundante; C: constância - (w) constante, (y) acessória; D: dominância - (d) dominante, (nd) não dominante, (sd) super dominante.

Tabela 6. Número médio de indivíduos coletados por área estudada em todo o período avaliado. Cuiabá-MT, 20042005.

Table 6. Average number of individuals collected by area studied in all period available. Brazil, Cuiabá, State of Mato Grosso, 2004-2005.

\begin{tabular}{lccc}
\multicolumn{1}{c}{ Espécie } & \multicolumn{3}{c}{ Área $^{\mathbf{1}}$} \\
\cline { 2 - 4 } & $\mathbf{1}$ & $\mathbf{2}$ & $\mathbf{3}$ \\
Anoplotermes sp. & $3 \pm 1,82^{\mathrm{fB}}$ & - & $74 \pm 8,61^{\mathrm{eA}}$ \\
Cornitermes silvestrii & $4 \pm 2,09^{\mathrm{fB}}$ & $160 \pm 12,67^{\mathrm{eA}}$ & - \\
Cornitermes villosus & - & $473 \pm 21,74^{\mathrm{dA}}$ & $1 \pm 1,08^{\mathrm{gB}}$ \\
Cylindrotermes parvignathus & - & $33 \pm 5,714^{\mathrm{F}}$ & - \\
Diversitermes diversimiles & $1023 \pm 31,99^{\mathrm{cB}}$ & $755 \pm 27,46^{\mathrm{cC}}$ & $1199 \pm 34,60^{\mathrm{cA}}$ \\
Heterotermes longiceps & - & - & $201 \pm 14,17^{\mathrm{D}}$ \\
Heterotermes tenuis & $4212 \pm 64,89^{\mathrm{aC}}$ & $9017 \pm 94,91^{\mathrm{aA}}$ & $4611 \pm 67,88^{\mathrm{aB}}$ \\
Nasutitermes kemneri & $1973 \pm 44,19^{\mathrm{bC}}$ & $5676 \pm 75,24^{\mathrm{bA}}$ & $2230 \pm 46,79^{\mathrm{bB}}$ \\
Parvitermes bacchanalis & $135 \pm 11,55^{\mathrm{eA}}$ & $38 \pm 6,01^{\mathrm{fB}}$ & $14 \pm 3,62^{\mathrm{fB}}$ \\
Ruptitermes sp. & - & $15 \pm 3,89^{\mathrm{F}}$ & - \\
Syntermes nanus & - & $111 \pm 10,56^{\mathrm{eA}}$ & $16 \pm 4,03^{\mathrm{fB}}$ \\
Velocitermes heteropterus & $432 \pm 20,74^{\mathrm{dB}}$ & $755 \pm 27,42^{\mathrm{cA}}$ & $119 \pm 10,63^{\mathrm{eC}}$ \\
Nasutitermitinae (Não ident.) & - & $179 \pm 13,29^{\mathrm{eA}}$ & $1 \pm 1,26^{\mathrm{gB}}$ \\
\hline
\end{tabular}

${ }^{1}$ Dados transformados $\sqrt{X+0,5}$. Médias seguidas de mesma letra minúscula nas colunas e maiúscula nas linhas não diferem entre si no nível de 5\% de probabilidade pelo teste Scott-Knott.

são favorecidas pelo fogo; entretanto, outras espécies tiveram sua abundância reduzida em áreas com histórico de queimadas trienais.

Em relação ao período de coleta, houve diferenças estatísticas entre espécies coletadas, sendo que o período de início das chuvas apresentou quantidade de indivíduos coletados significativamente maior, exceto para a espécie $D$. diversimiles, que teve seu maior pico no período seco (Tabela 7). De acordo com Constantino (1999), D. diversimiles são encontrados em abundância no Cerrado ocupando ninhos de outros cupins e, aparentemente, se alimentam de 
Tabela 7. Número médio de indivíduos coletados em três períodos de coletas nas três áreas de savana estudadas. Cuiabá-MT, 2004-2005.

Table 7. Average number of individuals collected in three periods in the three areas of savanna studied. Brazil, Cuiabá, State of Mato Grosso, 2004-2005.

\begin{tabular}{|c|c|c|c|}
\hline \multirow{2}{*}{ Espécie } & \multicolumn{3}{|c|}{ Coletas $^{1}$} \\
\hline & Seco & Início das chuvas & Chuvoso \\
\hline Anoplotermes sp. & $67 \pm 8,16^{\mathrm{gA}}$ & - & $10 \pm 3,23^{\mathrm{gB}}$ \\
\hline Cornitermes silvestrii & $2 \pm 1,60^{\mathrm{jB}}$ & $158 \pm 12,58^{\mathrm{fA}}$ & $4 \pm 2,13^{\mathrm{hB}}$ \\
\hline Cornitermes villosus & $125 \pm 11,18^{\mathrm{fB}}$ & $320 \pm 17,87^{\mathrm{dA}}$ & $29 \pm 5,44^{\mathrm{fC}}$ \\
\hline Cylindrotermes parvignathus & $33 \pm 5,73^{\mathrm{h}}$ & - & - \\
\hline Diversitermes diversimiles & $936 \pm 30,58^{\mathrm{cB}}$ & $401 \pm 20,03^{\mathrm{cC}}$ & $1641 \pm 40,50^{\mathrm{bA}}$ \\
\hline Heterotermes longiceps & $201 \pm 14,17^{e}$ & - & - \\
\hline Heterotermes tenuis & $5877 \pm 76,66^{\mathrm{aB}}$ & $8030 \pm 89,61^{\mathrm{aA}}$ & $3933 \pm 62,71^{\mathrm{aC}}$ \\
\hline Nasutitermes kemneri & $4471 \pm 66,86^{\mathrm{bB}}$ & $4956 \pm 70,40^{\mathrm{bA}}$ & $452 \pm 21,26^{\mathrm{cC}}$ \\
\hline Parvitermes bacchanalis & $125 \pm 11,18^{\mathrm{fA}}$ & $38 \pm 6,20^{\mathrm{hB}}$ & $25 \pm 5,03^{\mathrm{fC}}$ \\
\hline Ruptitermes sp. & $15 \pm 3,87^{\mathrm{i}}$ & - & - \\
\hline Syntermes nanus & $3 \pm 1,83^{\mathrm{jC}}$ & $77 \pm 8,78^{\mathrm{gA}}$ & $48 \pm 6,92^{\mathrm{eB}}$ \\
\hline Velocitermes heteropterus & $766 \pm 27,67^{\mathrm{dA}}$ & $339 \pm 18,42^{\mathrm{dB}}$ & $202 \pm 14,20^{\mathrm{dC}}$ \\
\hline Nasutitermitinae (Não ident.) & $1 \pm 1,32^{\mathrm{jB}}$ & $197 \pm 13,39^{\mathrm{eA}}$ & - \\
\hline
\end{tabular}

${ }^{1}$ Dados transformados $\sqrt{X+0,5}$. Médias seguidas de mesma letra minúscula nas colunas e maiúscula nas linhas não diferem entre si no nível de $5 \%$ de probabilidade pelo teste Scott-Knott.

madeira em decomposição; note-se que o período seco favorece a decomposição de material vegetal.

Junqueira et al. (2004), abordando a riqueza de espécies de cupins em área sazonal no estado de São Paulo, relataram que, na estação primavera, apresentou-se maior quantidade de indivíduos e de espécies coletadas. Não se pode fazer uma comparação detalhada com os resultados obtidos neste trabalho, pois, de acordo com Dorval et al. (2004), no município de Cuiabá, as estações do ano não são bem definidas, sendo de difícil caracterização e apresentando apenas dois períodos distintos: seco (maio-outubro) e chuvoso (novembro-abril); entretanto, a coleta que precede os meses da primavera acontece no período de início das chuvas (outubro), sendo que, nessa coleta, a ocorrência de cupins foi significativamente maior.

\section{CONCLUSÕES}

O período de seca e a má drenagem do solo propiciam condições para que seja registrada menor quantidade de cupins, sendo a área com dez anos sem fogo a mais rica em quantidade de espécies e de indivíduos.
As espécies Diversitermes diversimiles, Heterotermes tenuis, Nasutitermes kemneri, Parvitermes bacchanalis e Velocitermes heteropterus têm suas ocorrências independentemente do período. Diversamente, Cylindrotermes parvignathus, Ruptitermes sp., Heterotermes longiceps e Anoplotermes sp. podem ser consideradas espécies bioindicadoras de recuperação de áreas degradadas pelo fogo.

\section{AGRADECIMENTOS}

Ao Dr. Reginaldo Constantino, da Universidade Federal de Brasília - UNB, pela identificação das espécies, ao técnico Sr. Manoel Lauro da Silva, do Laboratório de Proteção Florestal, da Faculdade de Engenharia Florestal, pela inestimável ajuda no trabalho de campo, e ao Instituto de Linguística de Mato Grosso, pela cessão da área para instalação do experimento.

\section{STATUS DA SUBMISSÃO}

Recebido: 07/10/2011

Aceito: 12/02/2012

Resumo publicado online: 17/02/2012

Artigo completo publicado: 31/03/2012 


\section{AUTOR(ES) PARA CORRESPONDÊNCIA}

\section{Otávio Peres Filho}

Departamento de Engenharia Florestal, Universidade Federal de Mato Grosso - UFMT, Av. Fernando Corrêa da Costa, 2367, CEP 78125-070, Boa Esperança, Cuiabá, MT, Brasil e-mail: peres@ufmt.br

\author{
Alberto Dorval \\ Departamento de Engenharia Florestal, \\ Universidade Federal de Mato Grosso - UFMT, \\ Av. Fernando Corrêa da Costa, 2367, \\ CEP 78125-070, Boa Esperança, \\ Cuiabá, MT, Brasil \\ e-mail: adorval@terra.com.br
}

\section{REFERÊNCIAS}

Almeida JEM, Alves SB. Seleção de armadilhas para a captura de Heterotermes tenuis (Hagen). Anais da Sociedade Entomológica do Brasil 1995; 24(3): 619-624.

BRASIL. Ministério das Minas e Energia. Secretária Geral. Projeto RADAMBRASIL. Levantamentos dos Recursos Naturais. Rio de Janeiro, 1982. Folha SD 21 Cuiabá: geologia, geomorfologia, pedologia, vegetação e uso potencial da terra.

Campos MBS, Alves SB, Macedo N. Seleção de iscas celulósicas para o cupim Heterotermes tenuis (Isoptera: Rhinotermitidae) em cultura de cana-de-açúcar. Scientia Agricola 1998; 55(3): 480-484. http://dx.doi. org/10.1590/S0103-90161998000300017

Constantino R. Padrões de diversidade e endemismo de térmitas no bioma cerrado. In: Scariot AO, Silva JCS, Felfili JM, editors. Biodiversidade, Ecologia e Conservação do Cerrado. Brasília: Ministério do Meio Ambiente; 2005. p. 319-333.

Constantino R. Chave ilustrada para a identificação dos gêneros de cupins (Insecta: Isoptera) que ocorrem no Brasil. Papéis Avulsos de Zoologia 1999; 40(25): 387-448.

Coutinho LM. As queimadas e seu papel ecológico. Brasil Florestal. 1980; 10(10): 7-23.

Dias VLB. Impacto de fogo sobre cupins construtores de ninhos epígeos no cerrado [dissertação] Brasília: Universidade de Brasília; 1994.

Dorval A, Peres Filho O, Marques EN. Levantamento de Scolytidae (Coleoptera) em plantações de Eucalyptus spp. em Cuiabá, estado de Mato Grosso. Ciência Florestal. 2004; 14(1): 47-58.
Espírito-Santo Filho K. Efeito de distúrbios ambientais sobre a Fauna de cupins (Insecta: Isoptera) e seu papel como bioindicador [dissertação] Rio Claro: Universidade Estadual; 2005.

Ferreira DF. Sisvar: um programa para análises e ensino de estatística. Revista Symposium, 2008; 6(1): 36-41.

French JRJ, Robinson PJ, Bartlett NR. A rapid and relative field assessment of termite wood feeding preferences of the subterranean termite Heterotermes ferox (Frogg.) using toilet roll and small wood-block baits. Sociobiology, 1981; 6: 135-151.

Jones SC, Trosset MW, Nutting WL. Biotic and influences on foraging of Heterotermes aureus (Snyder) (Isoptera: Rhinotermitidae). Environment Entomology. 1987; 6: 791-795.

Junqueira LK, Dieh E, Florencio DF, Berti Filho E. Seasonal species richness of termite in an Eucalyptus plantation in Anhembi, State of São Paulo, Brazil. Acta Biologica Leopoldensia. 2004; 26(2): 241-248.

Lobry LAB, Conacher AJ. The role of termites and ants in soil modification: a review. Australian Journal of Soil Research. 1990; 28(1): 55-93.

Moino Junior A, Alves SB, Lopes RB, Neves PMOJ, Pereira RM, Vieira SA. External development of the entomopathogenic fungi Beauveria bassiana and Metarhizium anisopliae in the subterranean termite Heterotermes tenuis. Scientia Agricola. 2002; 59(2): 267-273. http://dx.doi.org/10.1590/S010390162002000200010

Moraes RCB, Haddad ML, Silveira Neto S, Reyes AEL. Software para análise estatística - ANAFAU. In: Anais do VIII Simpósio de Controle Biológico; 2003; São Pedro. Piracicaba: ESALQ/USP; 2003. p. 195.

Oliveira ARG, Freitas GA, Chaves EC, Lima Filho GF, Costa DA, Santos T, Crispim LS, Brandão D. Variação na Composição de Espécies e no Padrão de Riqueza e Abundância de Cupins ao Longo de uma Transição Cerrado - Campo Rupestre, no Morro Feio, Município de Hidrolândia, Goiás. In: Anais do VI Congresso de Ecologia do Brasil; 2003; Fortaleza. Fortaleza; 2003. cap. 3, p. 368-370.

Phillips TW. Responses of Hylastes salebrosus to turpentine, ethanol, and pheromones of Dendroctonus (Coleoptera: Scolytidae). The Florida Entomologist. 1990; 73(2): 286-292. http://dx.doi.org/10.2307/3494813

Sakagami SF, Laroca S. Observations on the bionomics of some neotropical Xylocopini bees, with some comparative biofaunistic notes (Hymenoptera, Anthophoridae). Journal of the Faculty of Science Hokkaido University, 1967; 18: 57-127.

Santos FM. Levantamento de cupins subterrâneos em plantios de Eucalyptus camaldulensis Dehnh. No município de Várzea Grande, estado de Mato Grosso 
[monografia] Cuiabá: Universidade Federal de Mato Grosso; 2004.

Santos MN, Teixeira MLF, Pereira MB, Menezes EB. Avaliação de estacas de Pinus sp. como iscaarmadilha em diversos períodos de exposição a cupins subterrâneos. Revista Floresta. 2010; 40(1): 29-36.

Sueta NY, Tamashiro M, Yates JR, Harverty M. Foraging behaviour of the Formosan subterranean termite
(Isoptera: Rhinotermitidae). Environment Entomology. 1984; 13: 1466-1470.

Vianna GMS, Carvalho SHC, Constantino R. Efeito do fogo sobre cupins subterrâneos (Isoptera) no Cerrado. In: Anais do XXV Congresso Brasileiro de Zoologia; 2004; Brasília. Brasília; 2004. p. 162. 